\title{
Relevancia del antecedente de absceso perirrenal en pacientes sometidos a nefrectomía simple retroperitoneal
}

\section{Relevance of Perirenal Abscess in Patients undergoing Retroperitoneal Simple Nephrectomy}

\author{
Luis Daniel Carrillo-Córdova ${ }^{1}$ Roberto Carlos Sarabia-Estrada ${ }^{1}$ Jean Manuel Jiménez-Villavicencio ${ }^{1}$ \\ Jhonatan Vitar-Sandoval ${ }^{1}$ Hugo Rivera-Astorga ${ }^{1}$ Gerardo Rodrigo Lemus-Mena ${ }^{1}$ \\ Eduardo Sánchez-Meza ${ }^{1}$ Jorge Raul Carrillo-Córdova ${ }^{2}$ Eli Jalil Camilo-Martínez ${ }^{1}$ \\ Miguel Angel Hernandez-Farias ${ }^{1}$ Alejandro Rosas-Ramírez ${ }^{1}$ José Francisco Virgen-Gutiérrez ${ }^{1}$ \\ Jorge Jaspersen-Gastelum ${ }^{1}$ Mateo Leopoldo Garduño-Arteaga ${ }^{1}$
}

\footnotetext{
${ }^{1}$ Departamento de Urología, Hospital General de México “Dr. Eduardo Liceaga," Ciudad de México, México

2 Departamento de Cirugía Plástica y Reconstructiva, Hospital General “Dr. Manuel Gea González," Ciudad de México, México
}

Address for correspondence Carrillo-Córdova Luis Daniel, MD, Cerro Teponaxtle \# 109, Colonia Campestre Churubusco, Delegación Coyoacan, Ciudad de México, México. CP: 04200

(e-mail: carrillocor@gmail.com).

\section{Resumen}

Palabras Clave

- nefrectomía

- absceso

- complicaciones

- exclusión renal

- litiasis
Antecedentes Las infecciones supurativas del riñón y del espacio peri nefrítico son poco comunes. La nefrectomía simple consiste en extraer el riñón contenido dentro de la fascia de Gerota, técnica usada principalmente para padecimientos no malignos del riñón. Ese término puede ser potencialmente engañoso ya que el término "simple" implica que la cirugía para enfermedad benigna, es técnicamente menos desafiante con menos complicaciones en relación a su contra parte: la nefrectomía radical.

Metodología Se analizaron 58 pacientes. Los parámetros demográficos de los pacientes con antecedente de absceso perirrenal, fueron comparados con los pacientes sin antecedente de absceso (Edad, género, peso, localización del lito) variables transoperatorias (tiempo quirúrgico, sangrado transoperatorio) y variables postquirúrgicas (necesidad de terapia intensiva, necesidad de transfusión, infección de herida quirúrgica y días de estancia hospitalaria).

Resultados Los pacientes se dividieron en dos grupos, los que contaban con antecedente de absceso (Grupo 1), y aquellos que no tenían antecedente de absceso (Grupo 2). Se encontraron veinte pacientes con antecedente de absceso $(34,55 \%)$ y treinta y ocho sin antecedente de absceso (65,6\%). Al realizar la comparación entre los dos grupos se encontraron con mayor frecuencia en el grupo diferencias estadísticamente significativas para las variables de tiempo quirúrgico (212 minuto (DE 46,6) vs 130.3 minuto (DE 49)p $=0.0001)$, días de estancia hospitalaria (8,6 días (DE 4,1 ) vs 5,6 días (DE 1.9) $p=0.0003$ ), e infección de herida quirúrgica (70\% vs $31 \% p=0,0008)$, no existieron diferencias estadísticamente significativas para el received

December 14, 2016

accepted

July 27, 2017

published online

July 10, 2018
DOI https://doi.org/ $10.1055 / \mathrm{s}-0038-1656555$. ISSN 0120-789X. eISSN 2027-0119.
Copyright $\odot$ 2018, Sociedad Colombiana License terms de Urología. Publicado por Thieme Revinter Publicações Ltda., Rio de Janeiro, Brazil. Todos los derechos reservados.

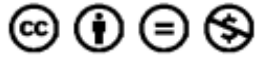


sangrado transoperatorio, necesidad de transfusión o necesidad de atención en unidad de cuidados intensivos.

Conclusiones La realización de una nefrectomía retroperitoneal simple abierta secundaria a litiasis en pacientes con antecedente de absceso perirrenal, conlleva a un mayor tiempo quirúrgico, secundario a una mayor dificultad técnica del procedimiento, por la gran cantidad de fibrosis y destrucción de la anatomía producida por el absceso. Los pacientes, también presentan un riesgo aumentado de infección de la herida quirúrgica, lo que conlleva a una mayor estancia hospitalaria y a una mayor inversión de recursos materiales y humanos.

Abstract

Keywords

- nephrectomy

- abscess

- nephrostomy

- complications

- non-functioning kidney

- lithiasis
Background Suppurative infections of the kidney and peri nephritic space are uncommon. Performing simple nephrectomy which is used to remove the kidney contained within the Gerota's fascia is used for non-malignant kidney disease. This term may be potentially misleading since the "simple" term implies that surgery for benign disease is technically less challenging with fewer complications relative to its radical nephrectomy counterpart.

Methodology 58 patients were analyzed. The demographic parameters of the patients with a history of perirenal abscess were compared with patients with out an antecedent abscess (age, gender, weight, calculi localization) transoperative variables (surgical time, transoperative bleeding) and postoperative variables (need for intensive care, need for Transfusion, surgical wound infection, and hospital stay days).

Results Patients were divided into two groups: abscess (Group 1) and those with no previous abscess (Group 2). Twenty patients with a history of abscess $(34.55 \%$ ) and thirty-eight with no history of an abscess (65.6\%) were found. Comparing the two groups were found more frequently in group 1 and statistically significant differences for the variables of surgical time (212 minute (DE 46.6) vs 130.3 minute (DE 49) $p=0.0001)$, days of hospital stay, And surgical wound infection $(70 \%$ vs $31 \%$ $p=0.0008)$, there were no statistically significant differences for operative bleeding, need for transfusion, or need for transfusion (8.6 days (SD 4.1) vs 5.6 days (SD 1.9) $p=0.0003$ ), or need for care in an intensive care unit.

Conclusions The performance of a simple open retroperitoneal nephrectomy secondary to lithiasis in patients with perirenal abscess, leads to a longer surgical time, secondary to a greater technical difficulty of the procedure, due to the great amount of fibrosis and destruction of the anatomy produced by The abscess. Patients are also at increased risk for surgical wound infection, leading to longer hospital stays and greater investment of material and human resources.

\section{Introducción}

Las infecciones supurativas del riñón y del espacio peri nefrítico son poco comunes, sin embargo, se asocian a una morbimortalidad elevada, afectando a ambos sexos por igual excepto los casos de absceso cortical que es 3 veces más común en el hombre que en la mujer. La incidencia se incrementa con la edad y habitualmente se asocia a uropatía obstructiva. Los abscesos renales representan $0,2 \%$ de todos los abscesos intraabdominales, de los cuales el $10 \%$ de los abscesos corticales rompen a través de la cápsula formando un absceso peri nefrítico, el cual es difícil de manejar y conlleva un peor pronóstico, con una alta mortalidad a pesar de tratamiento quirúrgico oportuno. ${ }^{1-3}$
Los pacientes que presentan antecedente de infecciones severas y abscesos renales con marcada destrucción de parenquima renal, pueden cursar con deterioro del funcionamiento renal secundario a infección crónica, recidivas de la litiasis, obstrucción y número de intervenciones quirúrgicas. A largo plazo, el paciente puede ser candidato a nefrectomía resolviendo definitivamente el proceso infeccioso. En casos más graves, el estado clínico puede progresar a insuficiencia renal y requerir diálisis, hemodiálisis o trasplante. ${ }^{4-6}$

La nefrectomía se reserva para los casos en los que existe un daño extenso y difuso del parénquima renal y en aquellos pacientes mayores en quienes la sobrevida depende de un manejo quirúrgico agresivo. 5,6 
La nefrectomía simple consiste en extraer el riñón contenido dentro de la fascia de Gerota, que es utilizada para patologías no malignas del riñón. Ese término puede ser potencialmente engañoso ya que el término "simple", implica que la cirugía para enfermedad benigna es técnicamente menos desafiante y con menos complicaciones en relación a su contraparte: la nefrectomía radical. Urólogos experimentados pueden mencionar que, en casos en donde se presenta inflamación difusa, así como cicatrices, pueden presentar un reto quirúrgico, siendo así que una nefrectomía para esos casos benignos puede no ser tan "simple."7

Las indicaciones de nefrectomía simple incluyen: riñón excluido o con pobre función como resultado de obstrucción, infección, trauma, litiasis, nefroesclerosis, reflujo vesico ureteral, riñón poliquístico o displasia congénita. La nefrectomía simple de un riñón funcional puede ser empleado para eliminar síntomas no tratables o problemas asociados, tales como es el sangrado, dolor, hipertensión, o infecciones persistentes. ${ }^{7-9}$

En esos casos donde se presenta inflamación, obstrucción crónica y presencia de fibrosis pueden generarse dificultades en la disección quirúrgica del riñón y requerir la habilidad de un cirujano experimentado. ${ }^{9-12}$

\section{Objetivo}

Describir los resultados de las nefrectomías simples retroperitoneales abiertas secundarias a litiasis realizadas en el Hospital General de México "Dr. Eduardo Liceaga” y determinar en los pacientes con antecedente de absceso perirrenal previo a la nefrectomía, las siguientes variables: tiempo quirúrgico, sangrado transoperatorio, complicaciones (de acuerdo a la clasificación de Clavien-Dindo), ${ }^{13}$ necesidad de transfusión, días de estancia hospitalaria, necesidad de terapia intensiva, e infección de herida quirúrgica comparada con los pacientes sin antecedente de absceso.

\section{Metodología}

Los datos utilizados para la elaboración de este estudio, se recabaron en el Hospital General de México "Dr. Eduardo Liceaga." Se buscaron expedientes en el archivo clínico que coincidieran con el código ICD-9 para nefrectomía, en el periodo comprendido de enero del 2010 a mayo del 2016. Se evaluó un total de 110 expedientes sometidos a nefrectomías abiertas. Se excluyeron 52 pacientes por no tener diagnóstico de litiasis. Se incluyeron un total de 58 pacientes con diagnóstico de exclusión renal confirmado mediante gamagrafía renal y litiasis. Los pacientes fueron sometidos a nefrectomía simple por un grupo de cirujanos expertos. En todos los pacientes se realizó un abordaje retroperitoneal por lumbotomía, y todos ellos estaban cubiertos con antibiótico al momento de la cirugía.

Se realizó un estudio descriptivo, trasversal, comparativo y prolectivo. Se dividieron los casos en dos grupos. El grupo 1, consistió en aquellos pacientes que tenían antecedente de haber presentado absceso perirrenal, el cual ya había sido drenado en un internamiento previo (mínimo 12 meses previos a la nefrectomía). El grupo 2, consistió en aquellos pacientes sometidos a nefrectomía, secundario a exclusión renal con litiasis, sin antecedente de absceso perirrenal.

Las variables demográficas de los pacientes del grupo 1 fueron comparadas con los pacientes del grupo 2 (edad, género, peso, localización del lito), variables transoperatorias (tiempo quirúrgico, sangrado transoperatorio) y variables postquirúrgicas (necesidad de terapia intensiva, necesidad de transfusión, infección de herida quirúrgica y días de estancia hospitalaria), mediante la $\mathrm{t}$ de student para las variables paramétricas y con una chi cuadrada con corrección de Yates para los datos no paramétricos.

\section{Resultados}

Se incluyeron un total de 58 pacientes con antecedente de nefrectomía simple abierta, de los cuales 24 pacientes correspondieron al género masculino $(41,4 \%)$ y 34 al género femenino (58,6\%). La media de edad fue de 46 años (DE 14,1). La localización del lito fue: piélico en $32 \%$, ureteral $6,9 \%$ y coral $55,2 \%$. Veinte pacientes tenían antecedente de absceso perirrenal (34,5\%). En cuanto a las variables transoperatorias, la media de sangrado transoperatorio fue de 604,9 mL (DE 720, Rango 20-5000 mL) y la media de tiempo quirúrgico, tomado desde la incisión en piel hasta el cierre de la herida fue de 158,6 minutos (DE 62, Rango 55-318). Las variables postoperatorias fueron las siguientes: diecinueve pacientes requirieron transfusión (32,8\%) (Clavien-Dindo II), diez pacientes presentaron lesión vascular o a órgano sólido durante la cirugía (17,2\%) (Clavien-Dindo III b) trece pacientes fueron admitidos en la unidad de terapia intensiva (22,4\%) (Clavien-Dindo IV), la media de estancia hospitalaria fue de 7 días (DE 2,3, Rango 2$10)$, veintidós $(37,9 \%)$ (Clavien-Dindo II), un paciente murió $(1,7 \%)$ (Clavien-Dindo V) (-Tabla 1).

Los pacientes se dividieron en dos grupos, pacientes con antecedente de absceso (Grupo 1) y aquellos que no tenían antecedente de absceso (Grupo 2). Se encontraron veinte pacientes con antecedente de absceso (34,55\%) y treinta y ocho sin antecedente de absceso (65,6\%) un paciente del Grupo 1 murió (Clavien-Dindo V) en el post operatorio inmediato. En la comparación de esos dos grupos se encontró una media de sangrado transoperatorio en el grupo 1 de $735 \mathrm{~mL}$ (DE 1050, rango 75-5000), y en el grupo 2 de $536 \mathrm{~mL}$ (DE 468, rango 20-1700), para tiempo quirúrgico en el grupo 1 la media fue de 212 minutos (DE 46.6, rango 100-318) y para el grupo 2 de 130,3 minutos (DE 49, rango 55-230), en cuanto a los días de estancia intrahospitalaria la media del grupo 1 fue de 8,6 días (DE 4,1, rango 4-15), y para el grupo 2 de 5,6 días (DE 1,9, rango 4-10), para necesidad de transfusión en el grupo 1,7 pacientes requirieron (35\%) y en el grupo 2, doce pacientes lo hicieron (31,6\%), del grupo 1 , seis pacientes requirieron cuidados en la unidad de cuidados intensivos (30\%), y en el grupo 2 , siete pacientes (18,4\%), en el grupo 1 en dos pacientes se documentó lesión vascular o a órgano (10\%) y en el grupo 2, en ocho pacientes también se presentó $(21,1 \%)$, 14 pacientes presentaron infección de herida quirúrgica en el 
Tabla 1 Estadística descriptiva para 58 pacientes posoperados de nefrectomía simple retroperitoneal secundaria a litiasis

\begin{tabular}{|c|c|c|c|}
\hline Variable & $(N)$ & Media & (\%) \\
\hline \multicolumn{4}{|l|}{ Genero } \\
\hline Masculino & 24 & $\mathrm{~N} / \mathrm{A}$ & $41,4 \%$ \\
\hline Femenino & 34 & $\mathrm{~N} / \mathrm{A}$ & $58,6 \%$ \\
\hline \multicolumn{4}{|l|}{ Localización del lito } \\
\hline Piélico & 19 & & $32,8 \%$ \\
\hline Ureteral & 4 & & $6,9 \%$ \\
\hline Coral & 32 & & $55,2 \%$ \\
\hline STO & 58 & $\begin{array}{l}609.9 \mathrm{~mL}(\mathrm{DE} 720, \\
\text { rango 20-5.000) }\end{array}$ & $N / A$ \\
\hline $\begin{array}{l}\text { Tiempo } \\
\text { quirúrgico }\end{array}$ & 58 & $\begin{array}{l}158.6 \text { minutos } \\
\text { (DE 62, } \\
\text { Rango 55-318) }\end{array}$ & $N / A$ \\
\hline Transfusión & 19 & $\mathrm{~N} / \mathrm{A}$ & $32,8 \%$ \\
\hline Lesión a órgano & 10 & $\mathrm{~N} / \mathrm{A}$ & $17,2 \%$ \\
\hline Necesidad de UTI & 13 & $\mathrm{~N} / \mathrm{A}$ & $22,4 \%$ \\
\hline $\mathrm{EIH}$ & 58 & $\begin{array}{l}7 \text { días (DE 2.3, } \\
\text { Rango } 2-10 \text { ) }\end{array}$ & $N / A$ \\
\hline Infección herida & 22 & $\mathrm{~N} / \mathrm{A}$ & $37,9 \%$ \\
\hline Muerte & 1 & $\mathrm{~N} / \mathrm{A}$ & $1,7 \%$ \\
\hline Nefrostomía & 20 & $\mathrm{~N} / \mathrm{A}$ & $34,5 \%$ \\
\hline
\end{tabular}

Abreviaturas: EIH, Estancia intrahospitalaria; STO, Sangrado transoperatorio; UTI, Unidad de cuidados intensivos.

Tabla 2 Comparación de las variables paramétricas en los dos grupos

\begin{tabular}{|l|l|l|l|}
\hline Variable & \multicolumn{2}{|l|}{ Media } & Valor de $\mathbf{p}$ \\
\hline & Grupo 1 & Grupo 2 & \\
\hline STO & $\begin{array}{l}735 \mathrm{~mL} \\
(\mathrm{DE} 1050)\end{array}$ & $\begin{array}{l}536 \mathrm{~mL} \\
\text { (DE 468) }\end{array}$ & $\mathbf{0 , 3 2 1 5}$ \\
\hline $\begin{array}{l}\text { Tiempo } \\
\text { Qx }\end{array}$ & $\begin{array}{l}212 \text { minutos } \\
\text { (DE 46.6) }\end{array}$ & $\begin{array}{l}130.3 \text { minutos } \\
\text { (DE 49) }\end{array}$ & $\mathbf{0 , 0 0 0 1 ^ { * }}$ \\
\hline ElH & $\begin{array}{l}8,6 \text { días } \\
(\mathrm{DE} \mathrm{4,1)}\end{array}$ & $\begin{array}{l}5,6 \text { días } \\
\text { (DE 1,9) }\end{array}$ & $\mathbf{0 , 0 0 0 3 ^ { * }}$ \\
\hline
\end{tabular}

Abreviaturas: EIH, Días de estancia hospitalaria; STO, Sangrado transoperatorio; tiempo Qx., tiempo quirúrgico de la incisión en piel al cierre de la herida.

*Diferencia estadísticamente significativa.

grupo 1 (70\%) y 8 pacientes (31\%) lo hicieron en el grupo 2 (-Tablas 2 y 3 ).

Al realizar la comparación entre los dos grupos, se encontraron diferencias estadísticamente significativas para las variables de tiempo quirúrgico $(p=0.0001)$, días de estancia hospitalaria $(p=0.0003)$, e infección de herida quirúrgica $(p=0.0008)$, no existieron diferencias estadísticamente significativas para el sangrado trasnoperatorio, necesidad de transfusión o necesidad de atención en unidad de cuidados intensivos.
Tabla 3 Comparación de las variables no paramétricas en los dos grupos

\begin{tabular}{|l|l|l|l|}
\hline Variable & & & Valor de $\mathrm{p}$ \\
\hline & $(\mathrm{N})$ y (\%) & & \\
\hline & Grupo 1 & Grupo 2 & \\
\hline Transfusión & $7(35 \%)$ & $12(31,6 \%)$ & 0,7919 \\
\hline UTI & $6(30 \%)$ & $7(18,4 \%)$ & 0,5004 \\
\hline LOVTO & $2(10 \%)$ & $8(21,1 \%)$ & 0,4880 \\
\hline IHQ & $14(70 \%)$ & $8(31 \%)$ & $\mathbf{0 , 0 0 0 8 ^ { * }}$ \\
\hline
\end{tabular}

Abreviaturas: IHQ, infección de herida quirúrgica; LOVTO, Lesión a órgano o lesión vascular en el transoperatorio; UTI, Necesidad de cuidado en unidad de cuidados intensivos.

*Diferencia estadísticamente significativa.

\section{Discusión}

Un porcentaje alto de pacientes con diagnóstico de exclusión renal que fueron sometidos a nefrectomía simple, presentaron antecedente de absceso perirrenal (34,5\%), los cuales habían sido tratados en internamiento previo mediante drenajes percutáneos e impregnación antibiótica, en todos los pacientes el absceso se presentó por lo menos 12 meses antes del procedimiento quirúrgico.

No se encontró una diferencia estadísticamente significativa en cuanto al sangrado transoperatorio, sin embargo, sí que existió en el tiempo quirúrgico ( $\mathrm{p}=0.0001$ ), atribuible a la pérdida de la anatomía y a la gran cantidad de fibrosis secundaria a la inflamación producida por absceso previo, lo cual hace de esas cirugías, un reto para el cirujano. No hay estudios que comparen esa asociación en la literatura médica.

Los pacientes con antecedente de absceso, presentaron tasas sumamente altas de infección de herida quirúrgica (IHQ) (70\%), al compararlas con los pacientes que no presentaron ese antecedente (31\%). Al realizar el análisis estadístico, se encontró una diferencia significativa $\left(0.0008^{*}\right)$.

Al compararlo con las tazas de IHQ reportadas de manera internacional, tenemos que la frecuencia de IHQ es difícil de monitorear porque los criterios para el diagnóstico no están estandarizados. Una encuesta patrocinada por la Organización Mundial de la Salud, demostró una prevalencia de infecciones nosocomiales en el rango del 3-21\%, con infecciones de la herida representando el 5-34\% del total. ${ }^{14}$

El informe de 2002 del Servicio Nacional de Vigilancia de Infecciones Nosocomiales de Inglaterra, ${ }^{15}$ que abarca el período comprendido entre octubre de 1997 y septiembre de 2001, indica que la incidencia de infecciones hospitalarias relacionadas con heridas quirúrgicas en el Reino Unido, es de hasta el $10 \%$ y cuesta al Servicio Nacional de Salud del país aproximadamente 1 billón de libras al año.

Eso muestra que los pacientes con antecedente de absceso, presentaron una incidencia de IHQ siete veces mayor que lo reportado en la literatura, mientras que los pacientes sometidos a nefrectomía por exclusión renal únicamente dos veces mayor. 
Un equipo estudió la asociación entre infección de herida quirúrgica en pacientes sometidos a nefrectomía con antecedente de colocación de nefrostomía, comparada a pacientes sin ese antecedente. ${ }^{16}$ Documentaron una serie de 31 pacientes y reportaron que el $77,7 \%$ de los pacientes con nefrostomía, presentaron infección de herida quirúrgica, mientras que en el grupo de pacientes sin catéter de nefrostomía únicamente el 31,8\%, encontrando una diferencia estadísticamente significativa. Concluyeron que los pacientes con antecedente de colocación de nefrostomía indicada por pionefrosis o para aliviar la obstrucción, que se sometieron a la nefrectomía simple debido a daño renal irrecuperable, tuvieron infecciones de heridas más tempranas y más frecuentes que los pacientes que se sometieron al mismo procedimiento sin nefrostomía.

La necesidad de transfusión y de cuidados especiales en una unidad de terapia intensiva fue altamente frecuente en los dos grupos; sin embargo, el tener antecedente de absceso no se asoció a un mayor riesgo de presentarlas. Los pacientes con antecedente de absceso, presentaron un promedio mayor y días de estancia hospitalaria, asociado a una mayor incidencia de infección de herida quirúrgica, requiriendo más días de tratamiento antibiótico.

\section{Conclusiones}

La realización de una nefrectomía retroperitoneal simple abierta secundaria a litiasis en pacientes con antecedente de absceso perirrenal, conlleva a un mayor tiempo quirúrgico, secundario a una mayor dificultad técnica del procedimiento por la gran cantidad de fibrosis y destrucción de la anatomía producida por el absceso.

Los pacientes, también están con un riesgo aumentado de presentar infección de la herida quirúrgica, lo que conlleva una mayor estancia hospitalaria y una mayor inversión de recursos materiales y humanos.

Este trabajo es la base para nuevos estudios que analicen la relación que existe entre el antecedente de absceso renal y las nuevas técnicas para realizar nefrectomías (laparoscopía y robot asistida).

\section{Responsabilidades Éticas}

Protección de personas y animales. Los autores declaran que los procedimientos seguidos, estuvieron a tono con las normas éticas del comité de experimentación humana responsable y de acuerdo con la Asociación Médica Mundial y la Declaración de Helsinki.

Confidencialidad de los datos. Los autores declaran que han seguido los protocolos de su centro de trabajo sobre la publicación de datos de pacientes.

Derecho a la privacidad y consentimiento informado. Los autores han obtenido el consentimiento informado de los pacientes y/o sujetos referidos en el artículo. Este documento obra en poder del autor de correspondencia.

Conflicto de Interés

Los autores de este trabajo reportan no tener ningún conflicto de interés.

\section{Bibliografía}

1 Dembry LM. Renal and Perinephric abscesses: Current treatment options. Infectious Diseases 2002;4:21-30

2 Meng MV, Mario LA, McAninch JW. Current treatment and outcomes of perinephric abscesses. J Urol 2002;168(4 Pt 1):1337-1340

3 Dembry LM, Andriole VT. Renal and perirenal abscesses. Infect Dis Clin North Am 1997;11(03):663-680

4 Yen DH, Hu SC, Tsai J, et al. Renal abscess: early diagnosis and treatment. Am J Emerg Med 1999;17(02):192-197

5 Rai RS, Karan SC, Kayastha A. Renal and Perinephric Abscesses Revisited. Med J Armed Forces India 2007;63(03):223-225

6 Jaik NP, Sajuitha K, Mathew M, et al. Renal abscess. J Assoc Physicians India 2006;54:241-243

7 Zelhof B, McIntyre IG, Fowler SM, Napier-Hemy RD, Burke DM, Grey BR; British Association of Urological Surgeons. Nephrectomy for benign disease in the UK: results from the British Association of Urological Surgeons nephrectomy database. BJU Int 2016;117 (01):138-144

8 Wein AJ, Kavoussi LR, Partin AW, et al. Campbell - Walsh Urology, Open Surgery of the Kidney, 11th edition, International Edition, Elsevier 2016 Cap. 60, pp. 1414-1445

9 Tepeler A, Akman T, Tok A, et al. Retroperitoneoscopic nephrectomy for non-functioning kidneys related to renal stone disease. Urol Res 2012;40(05):559-565

10 Raman JD, Sooriakumaran P, Cadeddu JA, Rane A. Radical and nonradical nephrectomy: no place for 'simple'. BJU Int 2009;103(07): 855-856

11 Connolly SS, O'Brien MF, Kunni IM, et al. Is simple nephrectomy truly simple? Comparison with the radical alternative. Ir J Med Sci 2011;180(01):177-179

12 Henderson JM, Fowler S, Joyce A, Dickinson A, Keeley FX; BAUS. Perioperative outcomes of 6042 nephrectomies in 2012: surgeonreported results in the UK from the British Association of Urological Surgeons (BAUS) nephrectomy database. BJU Int 2015;115(01):121-126

13 Dindo D, Demartines N, Clavien PA. Classification of surgical complications: a new proposal with evaluation in a cohort of 6336 patients and results of a survey. Ann Surg 2004;240(02): 205-213

14 Mayon-White RT, Ducel G, Kereselidze T, Tikomirov E. An international survey of the prevalence of hospital-acquired infection. J Hosp Infect 1988;11(Suppl A):43-48

15 Nosocomial infection National Surveillance Service (NINSS). Surgical site infection in English Hospitals: a national surveillance and quality improvement program. Public Health Laboratory Service. 2002

16 Greenstein A, Kaver I, Chen J, Matzkin H. Does preoperative nephrostomy increase the incidence of wound infection after nephrectomy? Urology 1999;53(01):50-52 